\title{
Stochastic Cutoff Method for Long-Range Interacting Systems
}

\author{
Munetaka Sasaki and Fumitaka Matsubara \\ Department of Applied Physics, Tohoku University, Sendai, 980-8579
}

(Received November 16, 2018)

\begin{abstract}
A new Monte Carlo method for long-range interacting systems is presented. This method involves eliminating interactions stochastically with the detailed balance condition satisfied. When pairwise interactions $V_{i j}$ of an $N$-particle system decrease with the distance as $r_{i j}^{-\alpha}$, computational time per Monte Carlo step is $\mathcal{O}(N)$ for $\alpha \geq d$ and $\mathcal{O}\left(N^{2-\alpha / d}\right)$ for $\alpha<d$, where $d$ is the spatial dimension. We apply the method to a two-dimensional magnetic dipolar system. The method enables us to treat a huge system of $256^{2}$ spins within a reasonable computational time, and reproduces a circular order originating from long-range dipolar interactions.
\end{abstract}

KEYWORDS: long-range interaction, Monte Carlo, algorithm, dipolar interactions 


\section{Introduction}

Numerical simulations of long-range interacting systems are quite difficult because we have to take a large number of interactions into consideration. If one carries out a naive Monte Carlo (MC) simulation of an $N$-particle system with pairwise interactions, computational time per MC step $t_{\mathrm{MC}}$ is proportional to $N^{2}$. Due to this rapid increase in computational time, accessible sizes for numerical simulations are restricted. One might think that this problem can be resolved by truncating interactions beyond a certain cutoff distance. However, such truncations often bring significant errors in various observables. ${ }^{1-6)}$ Concerning long-range interacting Ising ferromagnetism, a cluster algorithm that drastically improves computational efficiency without any approximation has been proposed.7) However, this method cannot be used for other systems. To overcome this difficulty, some approximate methods have been proposed until now. ${ }^{8-14)}$ Some of them can treat more than one million particles within a reasonable computational time with high accuracy. Furthermore, these methods are applicable to general long-range interacting systems. Nevertheless, these methods include some approximations more or less.

In this paper, we present a new MC method for general long-range interacting systems. In contrast to other methods, the present method is exact in the sense that it strictly satisfies the detailed balance condition. In this method, we stochastically switch long-range interactions $V_{i j}$ to either zero or a pseudointeraction $\bar{V}_{i j}$ by use of the stochastic potential switching algorithm. ${ }^{15,16)}$ Then the system is mapped on that with only $\bar{V}_{i j}$. The potential switching is performed every several steps. Since most of the distant (and weak) interactions are eliminated $\left(V_{i j} \rightarrow 0\right), t_{\mathrm{MC}}$ is significantly reduced. We refer to the present method as the stochastic cutoff (SCO) method. Of course, if one naively switches $V_{i j}$, it costs computational time $t_{\text {switch }}$ of order $N^{2}$. We develop an efficient method for the potential switching. In lattice systems, it reduces $t_{\text {switch }}$ to be comparable to $t_{\mathrm{MC}}$. We apply the SCO method to a two-dimensional magnetic dipolar system. By comparing our data with the previous ones, ${ }^{14)}$ we confirm that the SCO method gives correct results with modest computational time. We discuss the properties of the SCO method in comparison with other methods for long-range interacting systems.

The organization of the paper is as follows. In $\S 2$, we describe the SCO method. In $\S 3$, we show the results obtained by applying the SCO method to a two-dimensional magnetic dipolar system. In $\S 4$, we compare the SCO method with other methods. Section 5 is devoted to conclusions.

\section{Stochastic Cutoff (SCO) Method}

Before explaining the SCO method, we briefly survey the stochastic potential switching algorithm. ${ }^{15,16)}$ We hereafter consider a lattice system with pairwise long-range interactions 
described by the Hamiltonian

$$
\mathcal{H}=\sum_{i<j} V_{i j}\left(\boldsymbol{S}_{i}, \boldsymbol{S}_{j}\right)
$$

where $\boldsymbol{S}_{i}$ is a variable associated with the $i$-th element of the system. In this algorithm, $V_{i j}$ is stochastically switched to either $\tilde{V}_{i j}$ or $\bar{V}_{i j}$ with a probability of $P_{i j}$ or $1-P_{i j}$, respectively. The probability $P_{i j}$ is

$$
P_{i j}\left(\boldsymbol{S}_{i}, \boldsymbol{S}_{j}\right)=\exp \left[\beta\left(\Delta V_{i j}\left(\boldsymbol{S}_{i}, \boldsymbol{S}_{j}\right)-\Delta V_{i j}^{*}\right)\right]
$$

where $\beta$ is the inverse temperature, $\Delta V_{i j}\left(\boldsymbol{S}_{i}, \boldsymbol{S}_{j}\right) \equiv V_{i j}\left(\boldsymbol{S}_{i}, \boldsymbol{S}_{j}\right)-\tilde{V}_{i j}\left(\boldsymbol{S}_{i}, \boldsymbol{S}_{j}\right)$, and $\Delta V_{i j}^{*}$ is a constant equal to (or greater than) the maximum value of $\Delta V_{i j}\left(\boldsymbol{S}_{i}, \boldsymbol{S}_{j}\right)$ over all $\boldsymbol{S}_{i}$ and $\boldsymbol{S}_{j}$. We can choose the potential $\tilde{V}_{i j}$ arbitrarily. On the other hand, using $P_{i j}\left(\boldsymbol{S}_{i}, \boldsymbol{S}_{j}\right)$, the potential $\bar{V}_{i j}$ is given as

$$
\bar{V}_{i j}\left(\boldsymbol{S}_{i}, \boldsymbol{S}_{j}\right)=V_{i j}\left(\boldsymbol{S}_{i}, \boldsymbol{S}_{j}\right)-\beta^{-1} \log \left[1-P_{i j}\left(\boldsymbol{S}_{i}, \boldsymbol{S}_{j}\right)\right] .
$$

The arbitrariness of $\tilde{V}_{i j}$ can be utilized to reduce either the complexity of potential or cost to calculate it. With this potential switching process, the algorithm proceeds as follows:

(A) Potentials $V_{i j}$ are switched to either $\tilde{V}_{i j}$ or $\bar{V}_{i j}$ with the probability of $P_{i j}$ or $1-P_{i j}$, respectively.

(B) A standard MC simulation is performed with the switched Hamiltonian $\mathcal{H}^{\prime}$ expressed as

$$
\mathcal{H}^{\prime}=\sum_{i j}^{\prime} \tilde{V}_{i j}\left(\boldsymbol{S}_{i}, \boldsymbol{S}_{j}\right)+\sum_{i j}^{\prime \prime} \bar{V}_{i j}\left(\boldsymbol{S}_{i}, \boldsymbol{S}_{j}\right)
$$

where $\sum^{\prime}$ runs over all the potentials switched to $\tilde{V}$ and $\sum^{\prime \prime}$ runs over those switched to $\bar{V}$. The potential is fixed during the simulation.

(C) Return to (A).

It is shown that this MC procedure strictly satisfies the detailed balance condition with respect to the original Hamiltonian of eq. (1).

We give two remarks. Firstly, we can choose the period of the simulation in step (B) arbitrarily because the detailed balance condition is satisfied regardless of the period. Secondly, we do not need to switch all the potentials. For example, when the original Hamiltonian is $\mathcal{H}=\sum_{i j} V_{i j}+\sum_{i j} U_{i j}$, we can switch only $\left\{V_{i j}\right\}$ with $\left\{U_{i j}\right\}$ unswitched. Such partial switching is realized by setting $\tilde{U}_{i j}=U_{i j}$.

We now describe the SCO method. The basic idea is quite simple. We just set $\tilde{V}_{i j}=0$ to reduce computational time. In the following, we see that time $t_{\mathrm{MC}}$ in step (B) is significantly reduced since most potentials associated with distant interactions are switched to $\tilde{V}_{i j}=0$ in step (A). Now let us assume that interactions $V_{i j}$ decrease as $D r_{i j}^{-\alpha}(\alpha>0)$, where $D$ is a constant that represents the strength of interactions and $r_{i j}$ is the distance between sites $i$ and $j$. Systems with dipolar interactions correspond to the case $\alpha=3$. When we update an 
element from $\boldsymbol{S}_{i}^{(\text {old) }}$ to $\boldsymbol{S}_{i}^{(\text {new) }}$ in a MC simulation, we need to calculate the energy difference

$$
\Delta \mathcal{H}_{i}^{\prime} \equiv \sum_{k}^{\prime}\left[\bar{V}_{i k}\left(\boldsymbol{S}_{i}^{(\text {new })}, \boldsymbol{S}_{k}\right)-\bar{V}_{i k}\left(\boldsymbol{S}_{i}^{(\text {old })}, \boldsymbol{S}_{k}\right)\right]
$$

where the sum is taken over all the sites $k$ for which $V_{i k}$ is switched to $\bar{V}_{i k}$. This means that the time required to update $\boldsymbol{S}_{i}$ is proportional to the number of terms, i.e., $\mathcal{N}_{i} \equiv \sum_{k}^{\prime}$. When $r_{i k}$ is large, the probability that $V_{i k}$ is switched to $\bar{V}_{i k}$ is approximated as

$$
1-P_{i k} \sim D \beta r_{i k}^{-\alpha} \quad\left(r_{i k} \gg(D \beta)^{1 / \alpha}\right),
$$

where we have assumed that both $\Delta V_{i k}$ and $\Delta V_{i k}^{*}$ are of order $D r_{i k}^{-\alpha}$. Therefore, we can roughly evaluate $\mathcal{N}_{i}$ as

$$
\begin{aligned}
\mathcal{N}_{i} & \sim \int_{1}^{L} \mathrm{~d} r r^{d-1} D \beta r^{-\alpha} \\
& \sim\left\{\begin{array}{cc}
D \beta & (\alpha>d), \\
D \beta \log (L) & (\alpha=d), \\
D \beta L^{d-\alpha} & (\alpha<d),
\end{array}\right.
\end{aligned}
$$

where $d$ is the spatial dimension of the system, $L$ is the linear size of the lattice, and the lattice constant is assumed to be one. Therefore, computational time per one MC step $t_{\mathrm{MC}}$ is estimated as

$$
t_{\mathrm{MC}} \propto N \mathcal{N}_{i} \sim\left\{\begin{array}{cc}
D \beta N & (\alpha>d), \\
D \beta N \log (N) & (\alpha=d), \\
D \beta N^{2-\alpha / d} & (\alpha<d),
\end{array}\right.
$$

where $N\left(=L^{d}\right)$ is the total number of elements and the difference between $\log (L)$ and $\log (N)$ is ignored. Obviously, $t_{\mathrm{MC}}$ in the present method is much smaller than that in a naive MC method where $t_{\mathrm{MC}} \sim N^{2}$.

We next consider the potential switching process. If one switches $V_{i j}$ one by one, the potential switching time $t_{\text {switch }}$ is of order $N^{2}$. To reduce $t_{\text {switch }}$, we develop the following method. We first introduce a set of pairs $\left\{\mathcal{C}_{\text {pair }}(\boldsymbol{r})\right\}$ for which either $\boldsymbol{r}_{i j}$ or $\boldsymbol{r}_{j i}$ is $\boldsymbol{r}$, where $\boldsymbol{r}_{i j}$ is the vector spanning from sites $i$ to $j$. Figure 1 shows an example of such sets. Pairs are labeled sequentially. The point is that, in most cases, the probabilities of switching to $\bar{V}$ for $\left\{\mathcal{C}_{\text {pair }}(\boldsymbol{r})\right\}$ have some upper limit $p_{\max }(\boldsymbol{r})$. As we will show later, such maximum probability indeed exists in dipolar systems. Using $p_{\max }(\boldsymbol{r})$, we can switch the potentials in $\left\{\mathcal{C}_{\text {pair }}(\boldsymbol{r})\right\}$ in the following way:

a) Using $p_{\max }(\boldsymbol{r})$, we choose candidates that are switched to $\bar{V}_{i j}$. The potentials that are not chosen as a candidate are switched to 0 .

b) Switch each of the candidates to $\bar{V}_{i j}$ with the probability $\left[1-P_{i j}\left(\boldsymbol{S}_{i}, \boldsymbol{S}_{j}\right)\right] / p_{\max }(\boldsymbol{r})$. Otherwise, $V_{i j}$ is switched to 0 . 
Of course, if candidates are chosen one by one, it is very timeconsuming. We therefore choose them in the following way. We hereafter denote the potential of the $k$-th pair in $\left\{\mathcal{C}_{\text {pair }}(\boldsymbol{r})\right\}$ by $V^{(k)}$. Since the probability of being chosen as a candidate is the same in $\left\{\mathcal{C}_{\text {pair }}(\boldsymbol{r})\right\}$, the probability that $V^{(n)}$ is chosen as a candidate after $n-1$ successive failures is given by a geometric distribution $g\left(p_{\max }(\boldsymbol{r}), n\right)$, where

$$
g(p, n)=(1-p)^{n-1} p \quad(n \geq 1)
$$

An integer random variate $n$ that obeys $g(p, n)$ can be easily generated as

$$
n=\left\lceil\frac{\log (r)}{\log (1-p)}\right\rceil,
$$

where $\lceil x\rceil$ is the smallest integer that is greater than or equal to $x$, and $r$ is a continuous random variate with an uniform distribution of range $0<r \leq 1$. We can pick up only candidates by means of $g\left(p_{\max }(\boldsymbol{r}), n\right)$. For example, the generation of two random variates $n_{1}$ and $n_{2}$ means that there are only two candidates $V^{\left(n_{1}\right)}$ and $V^{\left(n_{1}+n_{2}\right)}$ among $n_{1}+n_{2}$ potentials. Using this idea, we have implemented the potential switching in $\left\{\mathcal{C}_{\text {pair }}(\boldsymbol{r})\right\}$ as follows:

1) Set $n_{\mathrm{s}}$ to be 0 , where $n_{s}$ is the number of potentials that have already been switched.

2) Generate an integer $n$ from the distribution $g\left(p_{\max }(\boldsymbol{r}), n\right)$ using eq. (10). If $n=1$, go to step 4). Otherwise, go to the next step.

3) Switch the $n-1$ potentials $\left(V^{\left(n_{\mathrm{s}}+1\right)}, V^{\left(n_{\mathrm{s}}+2\right)}, \cdots, V^{\left(n_{\mathrm{s}}+n-1\right)}\right)$ to 0 .

4) Switch $V^{\left(n_{\mathrm{s}}+n\right)}$ to $\bar{V}^{\left(n_{\mathrm{s}}+n\right)}$ with the probability $\left[1-P^{\left(n_{\mathrm{s}}+n\right)}\left(\boldsymbol{S}_{i}, \boldsymbol{S}_{j}\right)\right] / p_{\max }(\boldsymbol{r})$. Otherwise, switch it to 0 .

5) Finish the potential switching procedure if $n_{\mathrm{s}}+n$ is greater than (or equal to) the number of elements of $\left\{\mathcal{C}_{\text {pair }}(\boldsymbol{r})\right\}$. Otherwise, replace $n_{\mathrm{s}}$ with $n_{\mathrm{s}}+n$ and return to step 2).

Switching of all the potentials is completed by carrying out this procedure for all $\left\{\mathcal{C}_{\text {pair }}(\boldsymbol{r})\right\}$.

Now let us evaluate $t_{\text {switch. }}$. The potential switching process clearly requires time proportional to the number of potentials chosen as a candidate. To estimate it, we focus on the $N-1$ potentials associated with a certain site $i$ and estimate the number of candidates $\mathcal{N}_{i}^{(c)}$ among them. The total number of candidates is $N \mathcal{N}_{i}^{(c)}$. Since both $p_{\max }(\boldsymbol{r})$ and $1-P_{i k}$ are of order $D \beta r^{-\alpha}$ when $r \gg(D \beta)^{1 / \alpha}$, the order estimation of $\mathcal{N}_{i}^{(c)}$ and that of $\mathcal{N}_{i}$ given by eq. (7) are the same. We therefore obtain

$$
t_{\text {switch }} \sim\left\{\begin{array}{cc}
D \beta N & (\alpha>d), \\
D \beta N \log (N) & (\alpha=d), \\
D \beta N^{2-\alpha / d} & (\alpha<d) .
\end{array}\right.
$$

From eqs. (8) and (11), we find that $t_{\mathrm{switch}}$ is indeed comparable to $t_{\mathrm{MC}}$. 


\section{Results}

Now we apply the SCO method to a two-dimensional magnetic dipolar system on an $L \times L$ square lattice with open boundaries. The Hamiltonian of the system is described as

$$
\begin{aligned}
\mathcal{H}= & -J \sum_{\langle i j\rangle} \boldsymbol{S}_{i} \cdot \boldsymbol{S}_{j} \\
& +D \sum_{i<j}\left[\frac{\boldsymbol{S}_{i} \cdot \boldsymbol{S}_{j}}{r_{i j}^{3}}-3 \frac{\left(\boldsymbol{S}_{i} \cdot \boldsymbol{r}_{i j}\right)\left(\boldsymbol{S}_{j} \cdot \boldsymbol{r}_{i j}\right)}{r_{i j}^{5}}\right],
\end{aligned}
$$

where $\boldsymbol{S}_{i}$ is a classical Heisenberg spin of $\left|\boldsymbol{S}_{i}\right|=1,\langle i j\rangle$ runs over all the nearest-neighboring pairs, $\boldsymbol{r}_{i j}$ is the vector spanned from sites $i$ to $j$ in the unit of the lattice constant $a$, and $r_{i j}=\left|\boldsymbol{r}_{i j}\right|$. The first term describes short-range ferromagnetic exchange interactions and the second term describes long-range dipolar interactions, where $J(>0)$ is an exchange constant and $D=\left(g \mu_{\mathrm{B}} S\right) / a^{3}$. Hereafter, we regard $D$ as a parameter and consider the case that $D / J=0.1$. We choose this model as a benchmark of the SCO method because the properties of the model have been investigated extensively in previous work. ${ }^{14)}$ In particular, it is established that the model undergoes a phase transition from the paramagnetic state to a circularly ordered state at $T_{\mathrm{c}} \approx 0.88 \mathrm{~J}$ as a consequence of the cooperation of exchange and dipolar interactions.

Before showing the results, we explain the details of our simulation. We applied the SCO method only for dipolar interactions. The potential difference $\Delta V_{i j}$ is given as

$$
\Delta V_{i j}=V_{i j}=D\left[\frac{\boldsymbol{S}_{i} \cdot \boldsymbol{S}_{j}}{r_{i j}^{3}}-3 \frac{\left(\boldsymbol{S}_{i} \cdot \boldsymbol{r}_{i j}\right)\left(\boldsymbol{S}_{j} \cdot \boldsymbol{r}_{i j}\right)}{r_{i j}^{5}}\right] .
$$

It has the minimum value $-2 D / r_{i j}^{3}$ and the maximum value $+2 D / r_{i j}^{3}$ when $\boldsymbol{S}_{i}$ and $\boldsymbol{S}_{j}$ are parallel along $\boldsymbol{r}_{i j}$ and antiparallel, respectively. Therefore, we obtain

$$
\begin{aligned}
& p_{\max }(\boldsymbol{r}) \\
& =\max _{\boldsymbol{S}_{i}, \boldsymbol{S}_{j}}\left\{1-\exp \left[\beta\left(\Delta V_{i j}\left(\boldsymbol{S}_{i}, \boldsymbol{S}_{j}\right)-\Delta V_{i j}^{*}\right)\right]\right\} \\
& =1-\exp \left[-4 D \beta / r^{3}\right] \text {, }
\end{aligned}
$$

where we have set $\Delta V_{i j}^{*}=2 D / r^{3}$. The system was gradually cooled from an initial temperature $T=1.65 J$ to $0.05 J$ in steps of $\Delta T=0.05 J$. The initial temperature was set to be well above the critical temperature. The system was kept at each temperature for 100,000 MC steps, and potentials were switched for every $10 \mathrm{MC}$ steps. The first 50,000 MC steps are for equilibration and the following 50,000 MC steps are for measurement. Therefore, the total MC steps for one run is $3,300,000$. The computational time per run for $L=256$, i.e, the maximum size we examined, was less than three days when using a personal computer with a Core2Duo 2.40 $\mathrm{GHz}$ processor. We conducted simulations for 10 different runs with different initial conditions 
and random sequences. The energy was calculated for every $10 \mathrm{MC}$ steps with $\mathcal{O}(N \log N)$ computational time by utilizing the discrete Fourier convolution theorem and the fast Fourier transformation algorithm. For a detailed description of how the discrete Fourier convolution theorem is used for a system with open boundary conditions, we refer the reader to ref. 14 .

Now let us see the results of our simulations. Figure 2 shows the temperature dependences of the specific heat for different sizes. The peaks are located around $T_{\mathrm{c}} \approx 0.88 \mathrm{~J}$. In Fig. 3 , we show the spin structure observed at $T=0.05 \mathrm{~J}$. We clearly see a circular order that comes from long-range dipolar interactions. For a quantitative measurement of the circular order, we observed the absolute value of the circular component defined by

$$
M_{\phi} \equiv\left\langle\left|\left[\frac{1}{N} \sum_{i} \boldsymbol{S}_{i} \times \frac{\boldsymbol{r}_{i}-\boldsymbol{r}_{\mathrm{c}}}{\left|\boldsymbol{r}_{i}-\boldsymbol{r}_{\mathrm{c}}\right|}\right]\right|_{z}\right\rangle,
$$

where $\langle\cdots\rangle$ denotes the thermal average and $\boldsymbol{r}_{\mathrm{c}}$ is a vector describing the center of the lattice. Figure 4 shows the result. We see that the circular order rapidly grows around the critical temperature. We also performed a naive MC simulation for $L=48$ to confirm that the SCO method reproduces correct results. The crosses in Figs. 2 and 4 show the results.

Now let us examine the efficiency of the SCO method. Figure 5 shows the size dependences of the average computational time per MC step $t_{\mathrm{av}}$ for both the SCO method and the naive MC method. The average time $t_{\mathrm{av}}$ of the SCO method is given as

$$
t_{\mathrm{av}}=t_{\mathrm{MC}}+\frac{1}{10} t_{\mathrm{switch}}+\frac{1}{10} t_{\text {energy }}
$$

where $t_{\text {energy }}$ is the computational time per one energy measurement. Recall that potential switching and energy measurement are performed for every $10 \mathrm{MC}$ steps. We see that $t_{\mathrm{av}} \propto N$ in the SCO method, which is strongly in contrast with $t_{\mathrm{av}} \propto N^{2}$ in the naive MC method.

This huge reduction of the computational time in the SCO method comes from the reduction of interactions. We observed the average number $\bar{n}$ of potentials per site that survive as $\bar{V}_{i j}$. In Fig. 6, we show the temperature dependences of $\bar{n}$ for different sizes. It is impressive that $\bar{n}$ at each temperature converges to a certain value as the size increases. Although $\bar{n}$ increases with decreasing temperature, $\bar{n} \sim 22.5$ even at the lowest temperature.

In Fig. 7, we show the data of the specific heat measured in several simulations with different potential switching periods $N_{\mathrm{sw}}$. Since the system is kept at each temperature for $100,000 \mathrm{MC}$ steps, the number of potential switchings at each temperature is $100,000 / N_{\mathrm{sw}}$. We see that reliable results are obtained when $N_{\mathrm{sw}}$ is 1,000 or less. Figure 8 shows the size dependence of the ratio $t_{\mathrm{switch}} / t_{\mathrm{MC}}$. The ratio slightly depends on the size, and it is 1.65 at most. This means that we can even switch potentials at every MC step with a reasonable cost.

Lastly, we compare relaxation speeds between the SCO method and the naive MC method. Figure 9 shows the time evolution of $M_{\phi}$ for $L=48$ when the system is kept at $T=0.4 J$. As shown in the inset, the relaxation speed of the SCO method is about 1.4 times slower than 
J. Phys. Soc. Jpn.

Full PAPER

that of the naive MC method. We have also performed a similar measurement at $T=0.7 J$, and found that the ratio is about 1.2. It is clear from Fig. 5 that the relaxation speed of the SCO method is much higher than that of the naive MC method if they are compared in terms of the computational time.

\section{Comparison of the SCO Method with Other Methods}

In this section, we discuss the properties of the SCO method in comparison with those of other methods for long-range interacting systems. As we have emphasized, the primary merit of the SCO method is that it involves no approximation. The performance of this method strongly depends on the conditions of simulations in terms of parameters such as the temperature $T$, the spatial dimension $d$, the decay exponent of potentials $\alpha$, and the strength of potentials $D$, as illustrated by eqs. (8) and (11). Since $t_{\mathrm{MC}}$ and $t_{\mathrm{switch}}$ are proportional to $D \beta$, the SCO method is particularly efficient for systems with strong short-range interactions and weak long-range interactions. The reason is as follows: Since the system is dominated by short-range interactions, we expect $k_{\mathrm{B}} T_{\mathrm{c}} \sim D_{\mathrm{SR}}$, where $D_{\mathrm{SR}}$ is the strength of short-range interactions. This means that $t_{\mathrm{MC}}$ and $t_{\mathrm{switch}}$ are very small around the critical temperature because

$$
D \beta_{\mathrm{c}} \sim D / D_{\mathrm{SR}} \ll 1 .
$$

In this sense, the SCO method is really suitable for magnetic dipolar systems, as we have

demonstrated. In magnetic dipolar systems, the ratio $J / D$ is usually on the order of hundreds or even thousands. On the other hand, when $\alpha<d$, the computational time in the SCO method is $\mathcal{O}\left(N^{2-\alpha / d}\right)$. The SCO method is less efficient in such a case because the computational time is of order $N$ or $N \log N$ in most of the other methods.

Lastly, we emphasize that the SCO method can be made applicable to off-lattice systems by developing some efficient method for potential switching.

\section{Conclusion Remarks}

In the present work, we have proposed a new MC method based on the stochastic potential switching algorithm. ${ }^{15,16)}$ To our knowledge, this is the first method for general long-range interacting systems that greatly reduces computational time without any approximation. This method is applicable to any lattice system with long-range interactions. The efficiency of the SCO method has been demonstrated by applying it to a two-dimensional magnetic dipolar system. We have also discussed the properties of the SCO method in comparison with those of other methods for long-range interacting systems.

\section{Acknowledgment}

This work is supported by a Grant-in-Aid for Scientific Research (\#18740226) from MEXT in Japan. 


\section{References}

1) C. L. Brooks III, B. M. Pettitt, and M. Karplus: J. Chem. Phys. 83 (1985) 5897.

2) R. J. Loncharich and B. R. Brooks: Proteins 6 (1989) 32.

3) H. Schreiber and O. Steinhauser: Chem. Phys. 168 (1992) 75.

4) J. Guenot and P. Kollman: J. Comput. Chem. 14 (1993) 295.

5) P. J. Steinbach and B. R. Brooks: J. Comput. Chem. 15 (1994) 667.

6) M. Saito: J. Chem. Phys. 101 (1994) 4055.

7) E. Luijten and H. W. J. Blöte: Int. J. Mod. Phys. C 6 (1995) 359.

8) A. W. Appel: SIAM J. Sci. Stat. Comput. 6 (1985) 85.

9) J. Barnes and P. Hut: Nature 324 (1986) 446.

10) L. Greengard: The Rapid Evolution of Potential Fields in Particle Systems (MIT Press, Cambridge, MA, 1988).

11) J. Carrier, L. Greengard, and V. Rokhlin: SIAM J. Sci. Stat. Comput. 9 (1988) 669.

12) M. Saito: Mol. Simul. 8 (1992) 321.

13) H.-Q. Ding, N. Karasawa, and W. A. Goddard III: J. Chem. Phys. 97 (1992) 4309.

14) J. Sasaki and F. Matsubara: J. Phys. Soc. Jpn. 66 (1996) 2138.

15) C. H. Mak: J. Chem. Phys. 122 (2005) 214110.

16) C. H. Mak and A. K. Sharma: Phys. Rev. Lett. 98 (2007) 180602. 


\section{Figure Captions}

Figure 1 : Set of pairs $\left\{\mathcal{C}_{\text {pair }}(\boldsymbol{r})\right\}(\boldsymbol{r}=(1,1))$ and their labels in a $5 \times 5$ square lattice. The set consists of 16 elements.

Figure 2: (Color online) Temperature dependences of the specific heat $C$ for different sizes. The average is taken over 10 different runs.

Figure 3 : Snapshot of the spin structure at $T=0.05 J$ on a $48 \times 48$ square lattice obtained by the SCO method.

Figure 4 : (Color online) Temperature dependences of the circular component of the magnetization $M_{\phi}$ for different sizes. The average is taken over 10 different runs.

Figure 5 : (Color online) Average computational time per MC step $t_{\mathrm{av}}$ is plotted as a function of $N=L^{2}$ for the SCO method (full squares) and a naive MC method (full circles). The solid line and dashed line are proportional to $x$ and $x^{2}$, respectively.

Figure 6 : (Color online) Temperature dependences of the average number $\bar{n}$ of potentials $\left(\bar{V}_{i j} \neq 0\right)$ per site. The average is taken over 10 different runs.

Figure 7 : (Color online) Temperature dependences of the specific heat on a $48 \times 48$ square lattice with different $N_{\mathrm{sw}}$ values, where $N_{\mathrm{sw}}$ is the number of MC steps for every which potentials are switched. The data measured in a single run are shown.

Figure 8 : Ratio $t_{\mathrm{switch}} / t_{\mathrm{MC}}$ is plotted as a function of $N=L^{2}$.

Figure $9:$ (Color online) Time evolution of the circular component of the magnetization $M_{\phi}$ for $L=48$ when the system is kept at $T=0.4 J$. The average is taken over 1,000 different runs. The data of the SCO method are denoted by crosses (below) and those of the naive MC method are denoted by open squares (above). In the inset, $M_{\phi}$ of the SCO method and that of the naive MC method are plotted as a function of $t_{\mathrm{s}}=t / 1.4$ and $t$, respectively. 


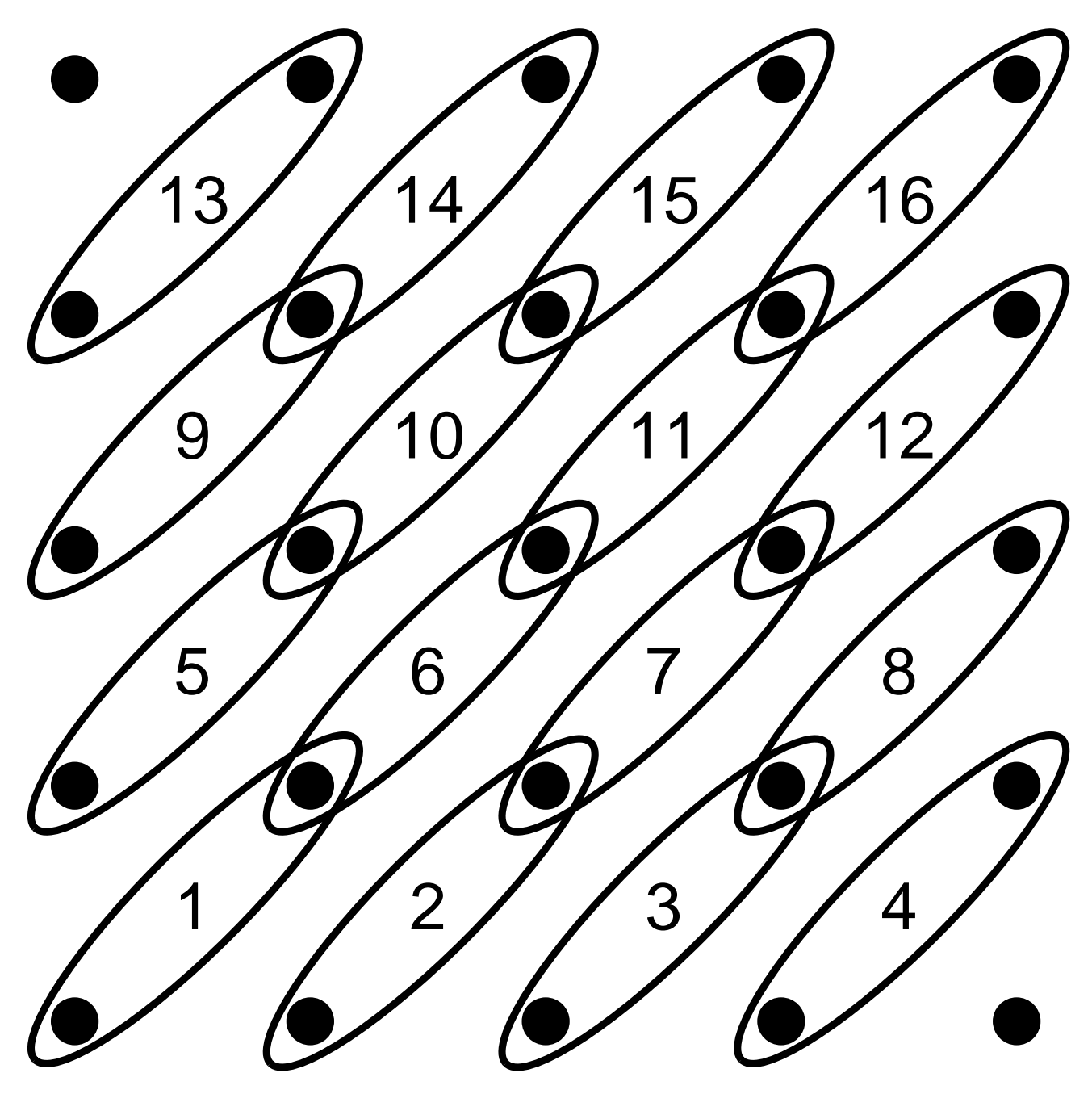

Fig. 1. 


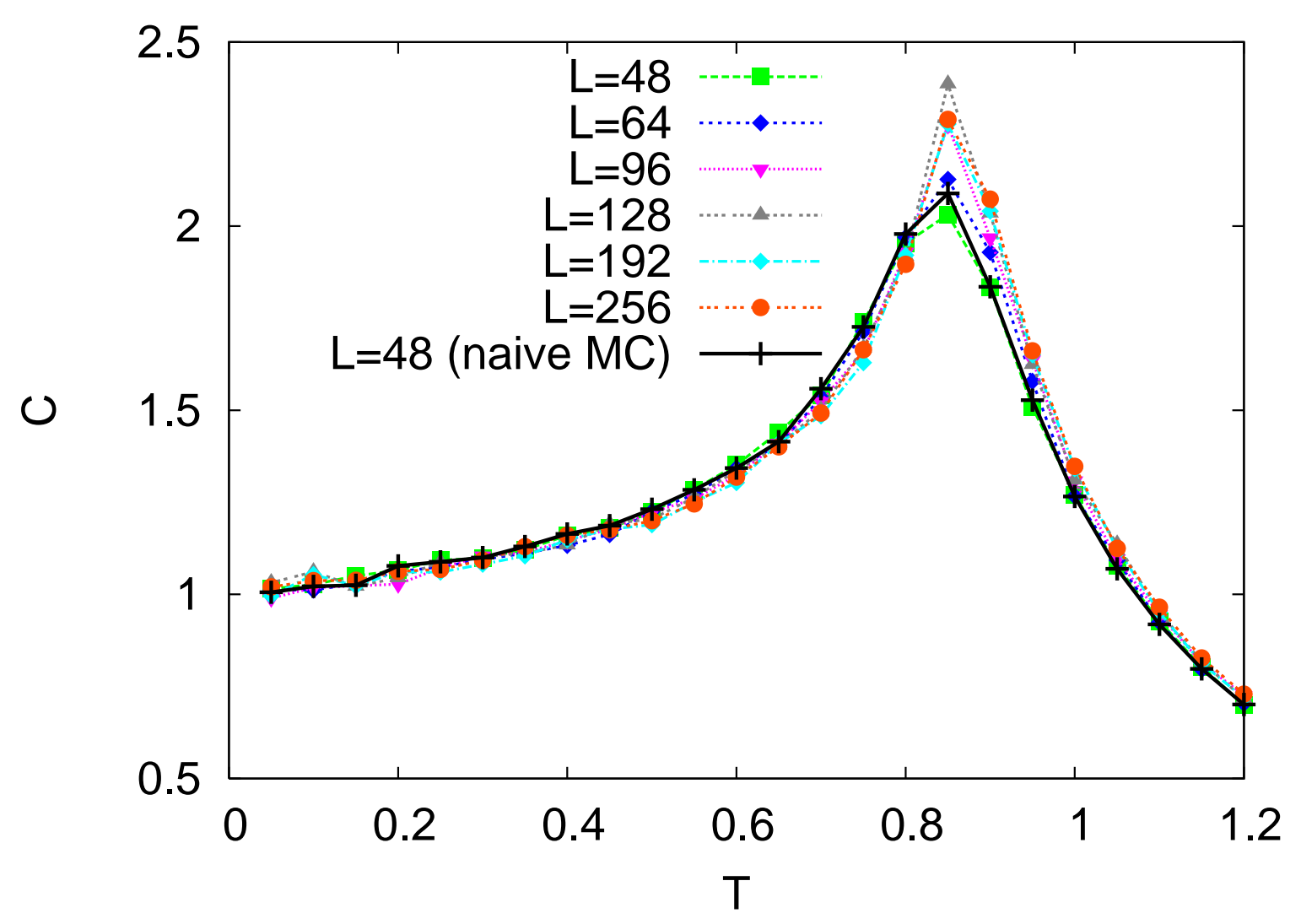

Fig. 2. 


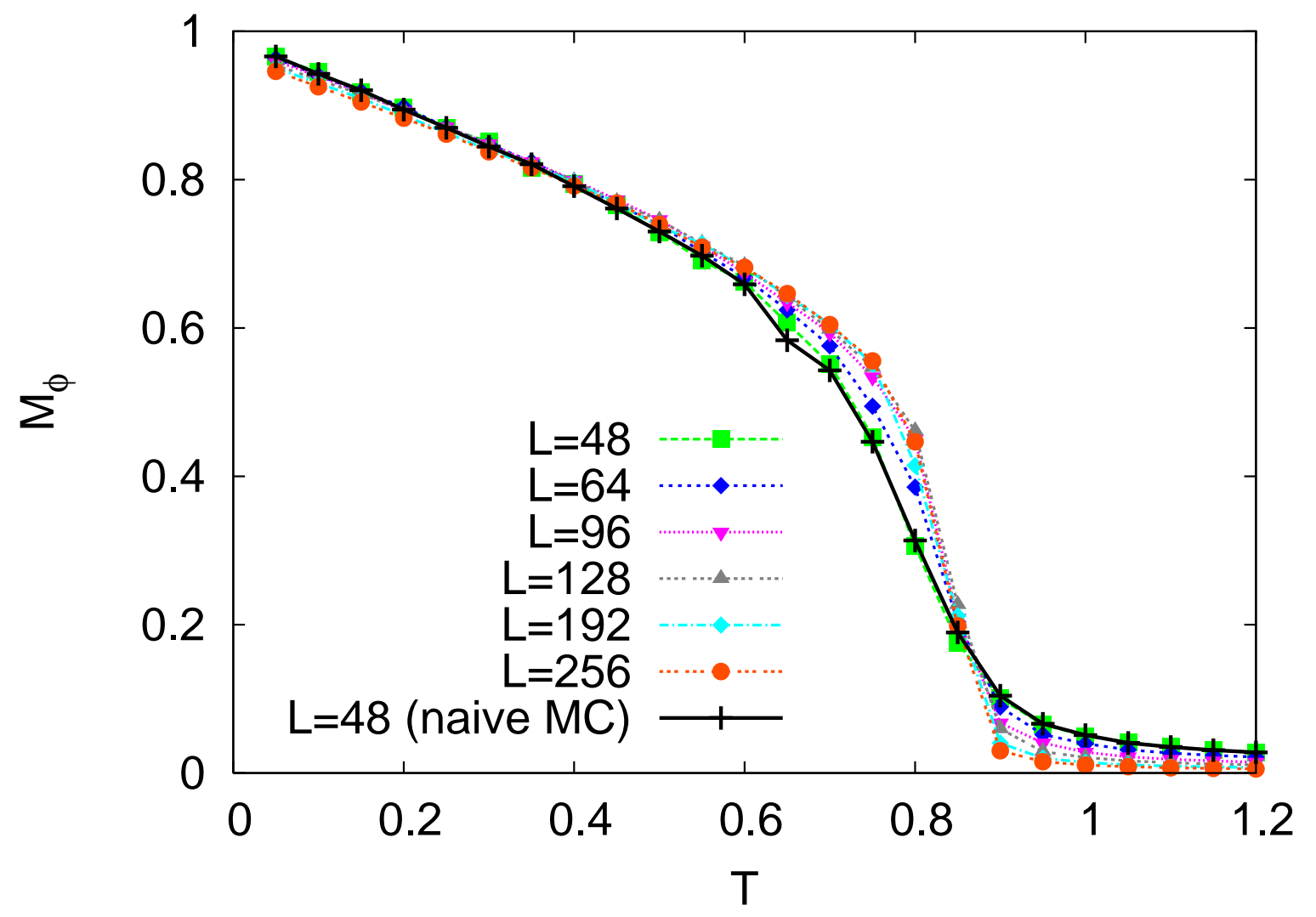

Fig. 4. 


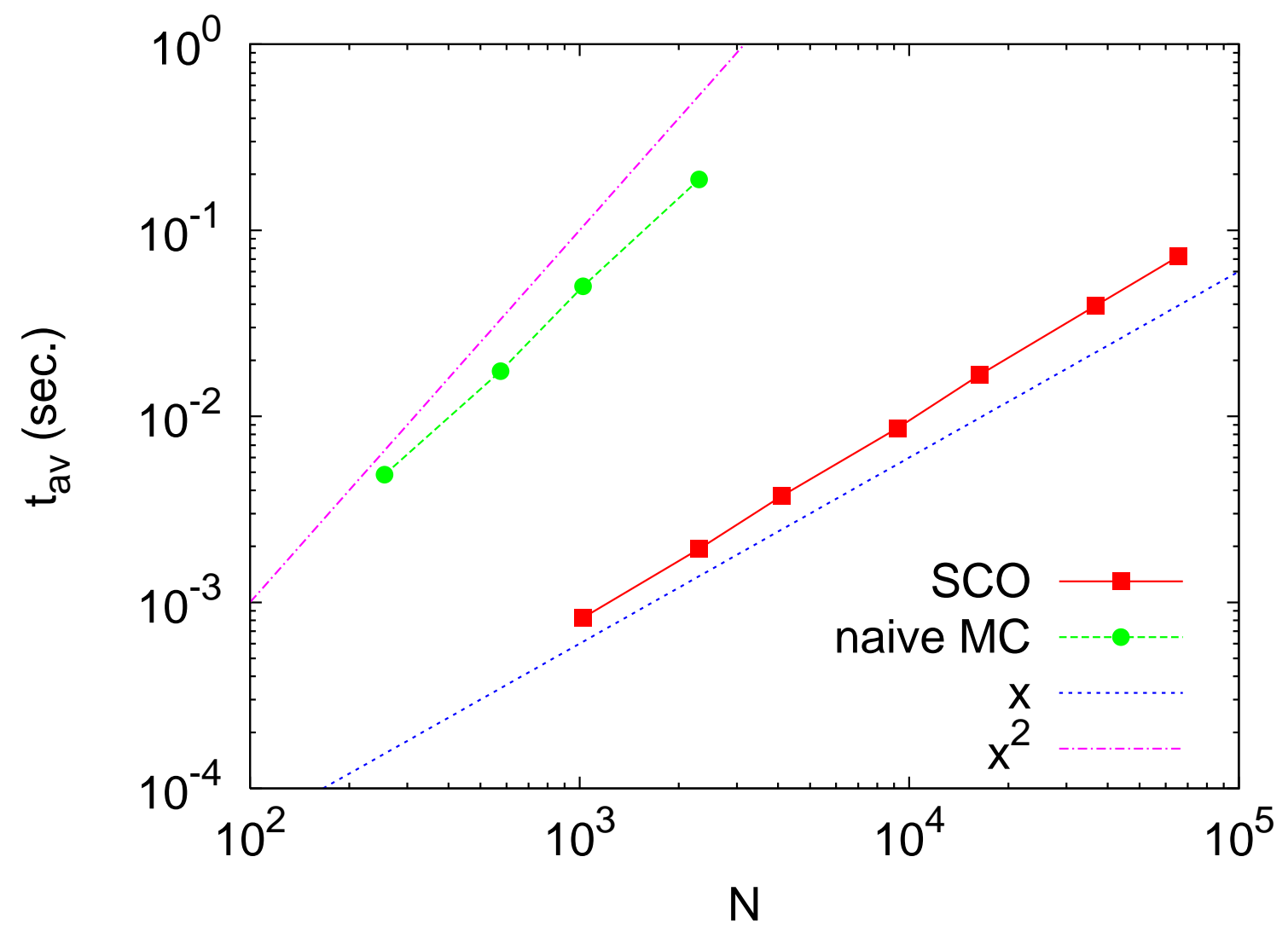

Fig. 5. 


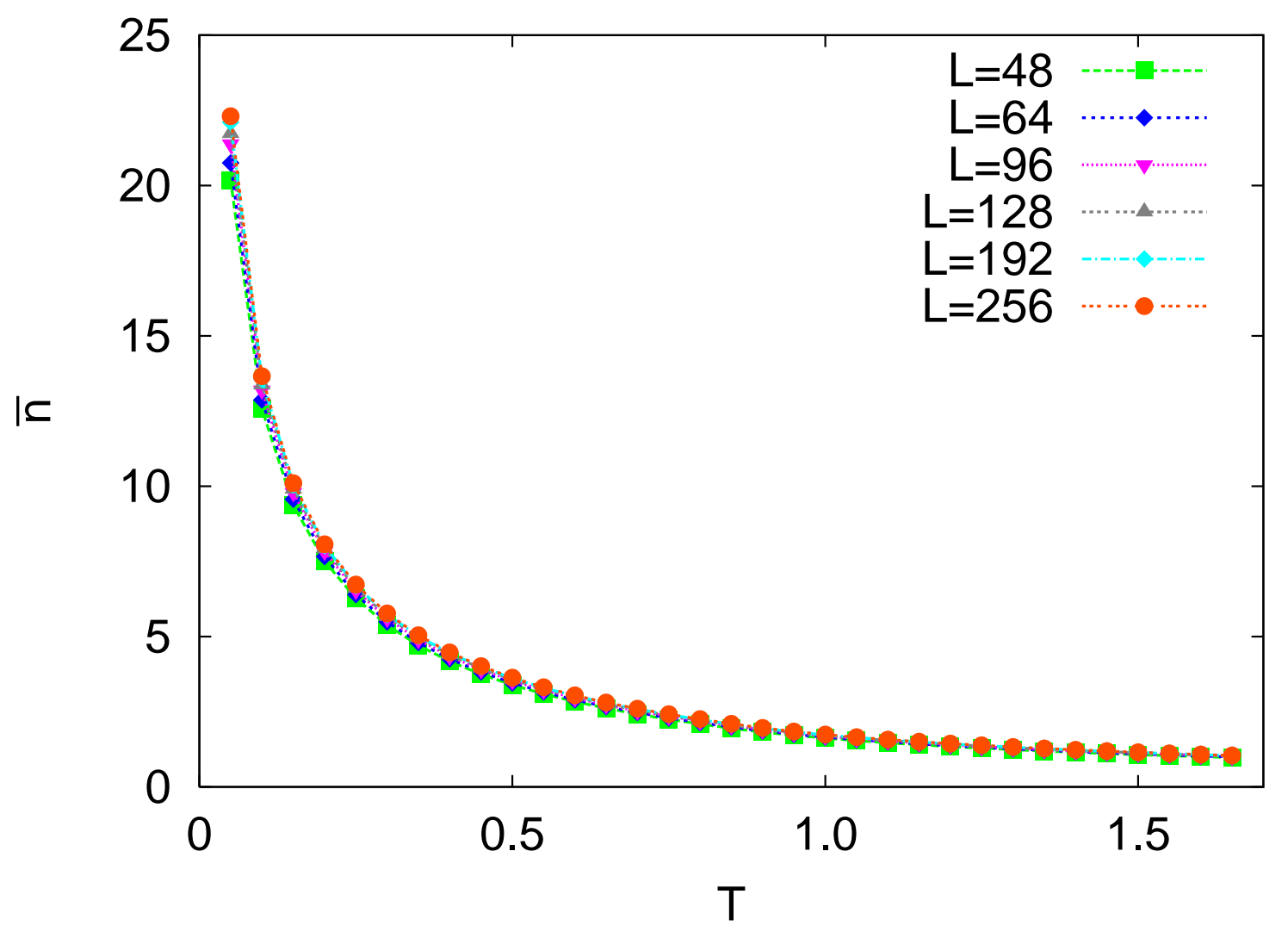

Fig. 6. 


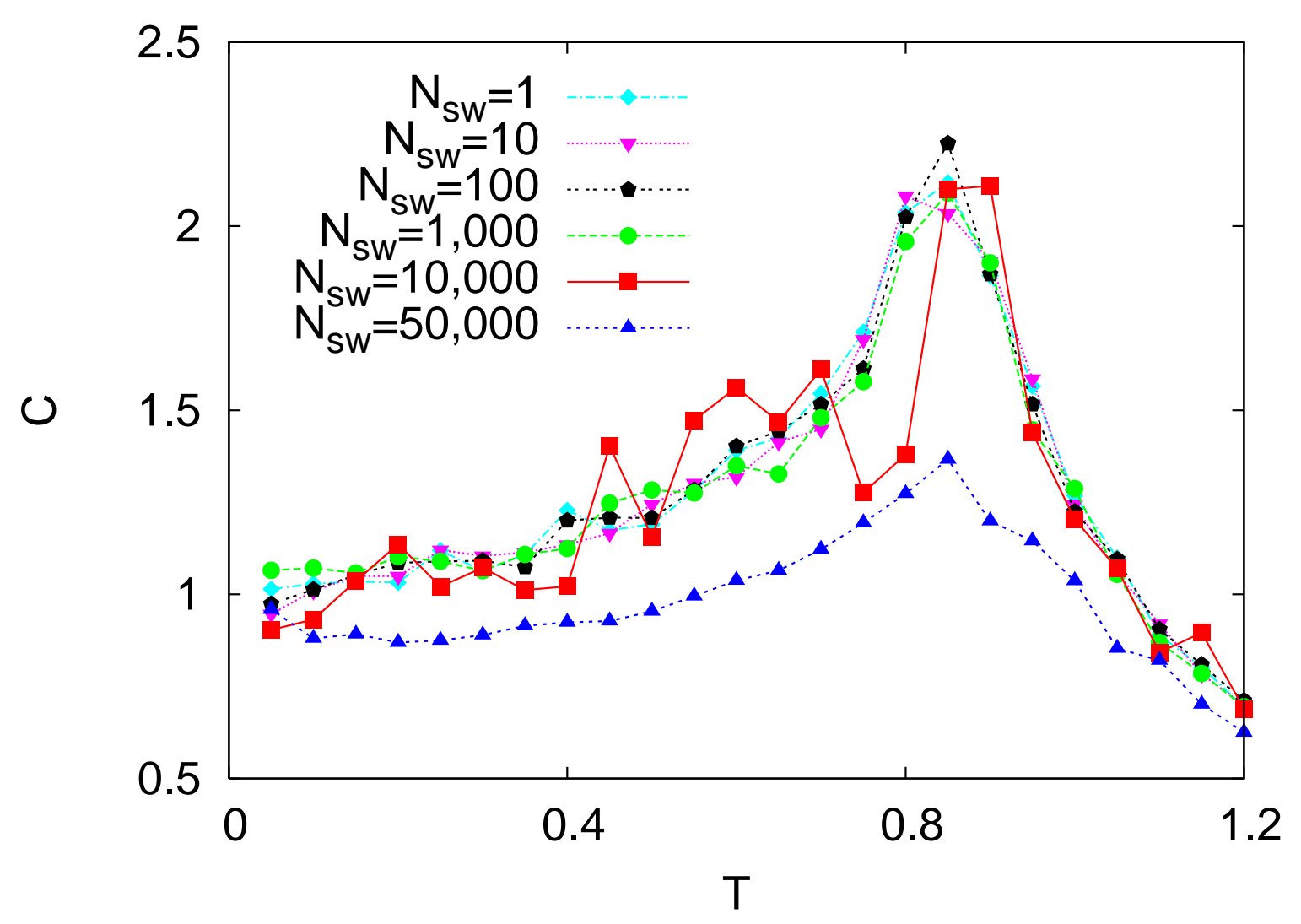

Fig. 7. 


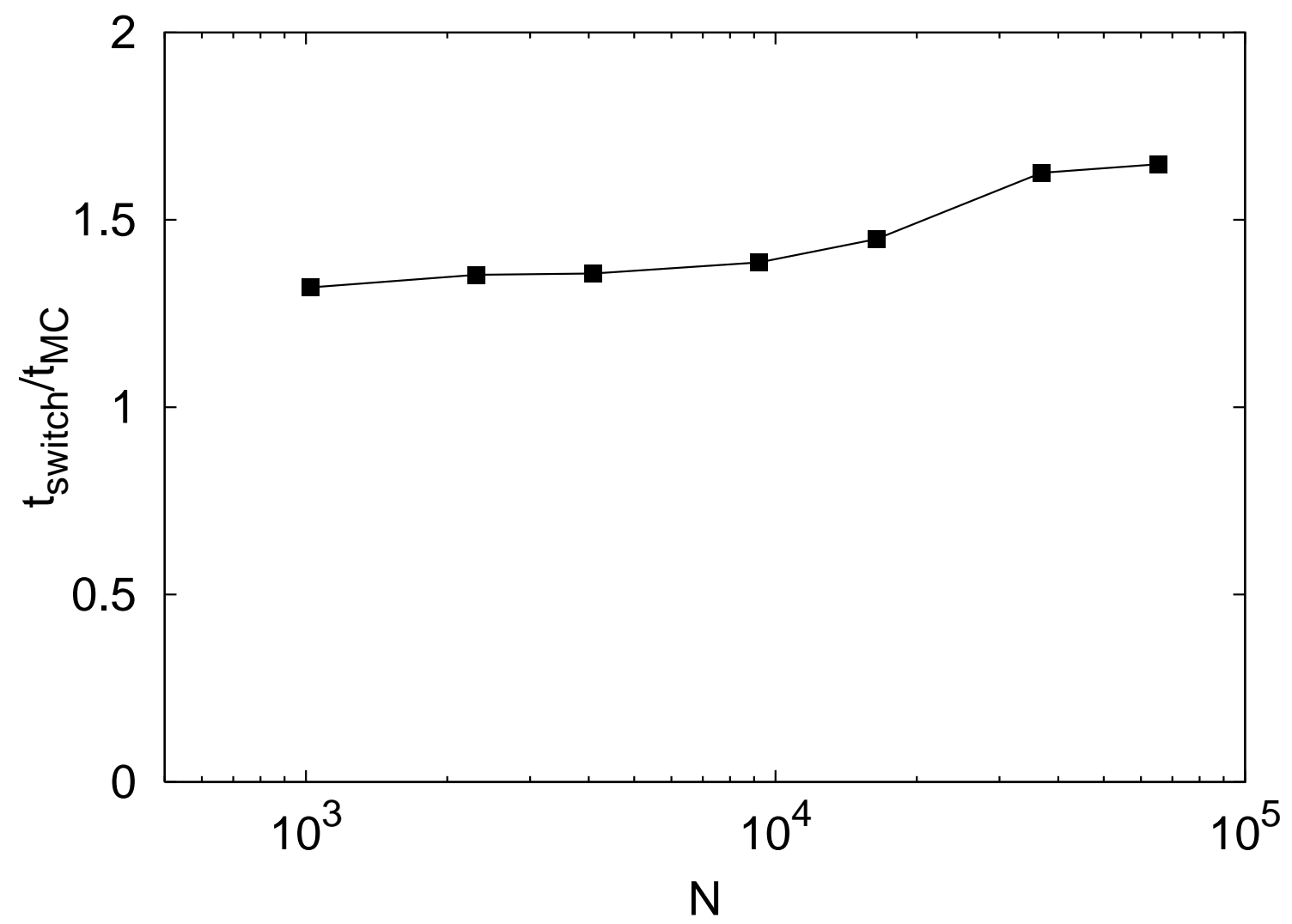

Fig. 8. 


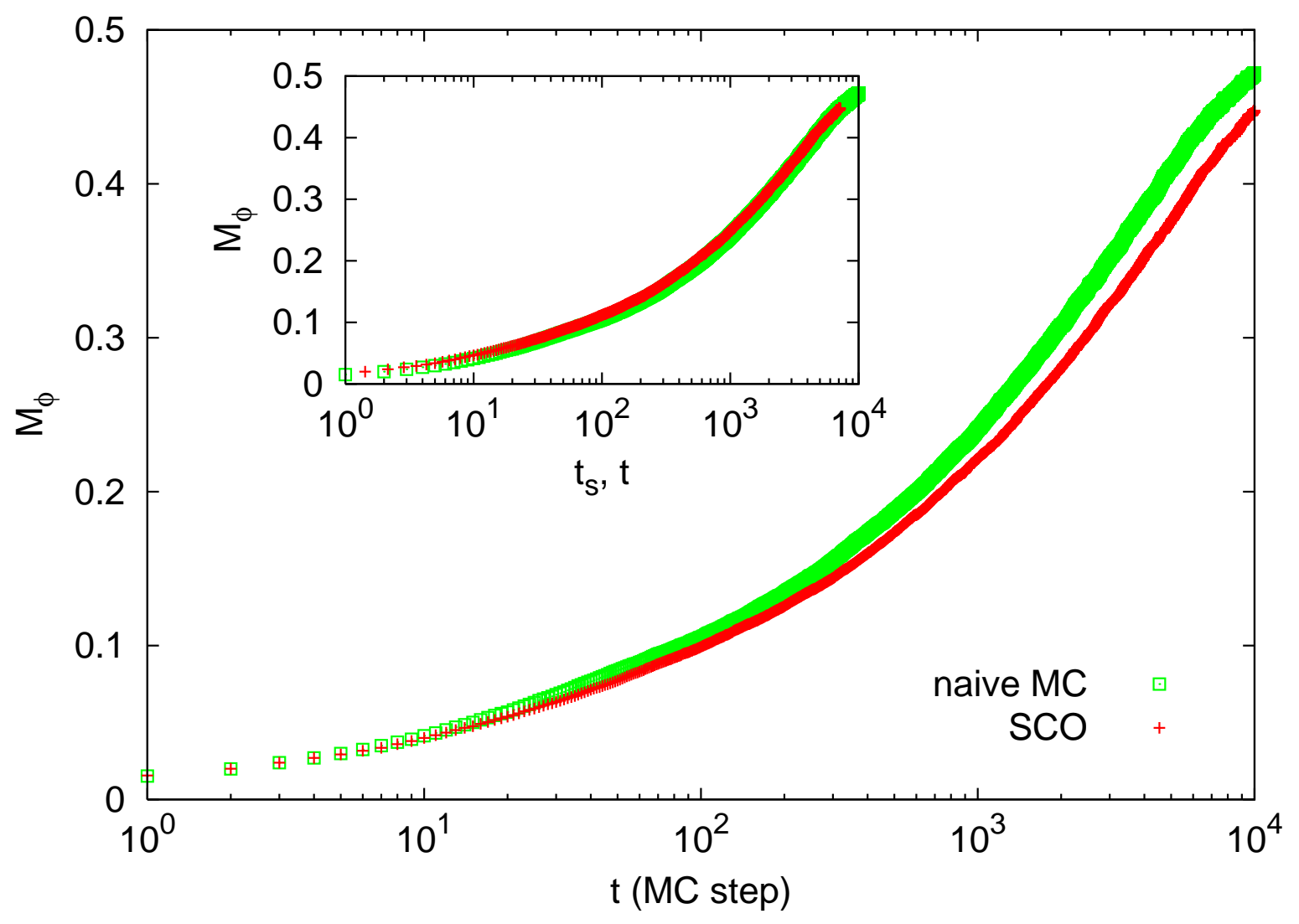

Fig. 9. 